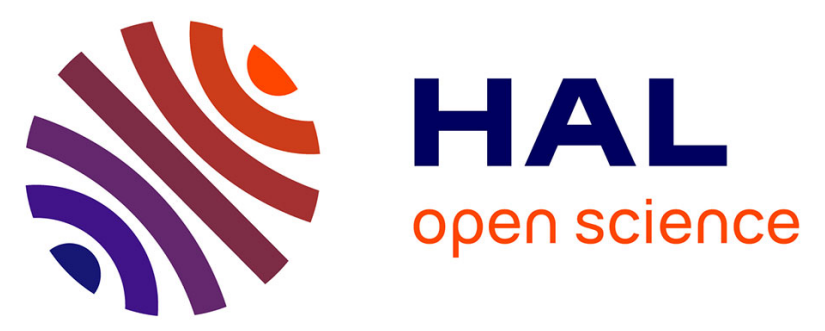

\title{
The genome of Geosiphon pyriformis reveals ancestral traits linked to the emergence of the arbuscular mycorrhizal symbiosis
}

\author{
Mathu Malar C, Manuela Krüger, Claudia Krüger, Yan Wang, Jason E \\ Stajich, Jean Keller, Eric C H Chen, Gokalp Yildirir, Matthew \\ Villeneuve-Laroche, Christophe Roux, et al.
}

\section{To cite this version:}

Mathu Malar C, Manuela Krüger, Claudia Krüger, Yan Wang, Jason E Stajich, et al.. The genome of Geosiphon pyriformis reveals ancestral traits linked to the emergence of the arbuscular mycorrhizal symbiosis. Current Biology - CB, 2021, 31 (7), pp.1570-1577.e4. 10.1016/j.cub.2021.01.058 . hal03327905

\section{HAL Id: hal-03327905 \\ https://hal.science/hal-03327905}

Submitted on 1 Sep 2021

HAL is a multi-disciplinary open access archive for the deposit and dissemination of scientific research documents, whether they are published or not. The documents may come from teaching and research institutions in France or abroad, or from public or private research centers.
L'archive ouverte pluridisciplinaire HAL, est destinée au dépôt et à la diffusion de documents scientifiques de niveau recherche, publiés ou non, émanant des établissements d'enseignement et de recherche français ou étrangers, des laboratoires publics ou privés. 
1 The genome of Geosiphon pyriformis reveals ancestral traits linked to the

2 emergence of the arbuscular mycorrhizal symbiosis

3 Mathu Malar $\mathrm{C}^{1^{*}}$, Manuela Krüger ${ }^{2,3,{ }^{*}}$, Claudia Krüger ${ }^{2,3^{*}}$, Yan Wang ${ }^{4,5}$, Jason E. Stajich ${ }^{6}$,

4 Jean Keller ${ }^{7}$, Eric C.H. Chen ${ }^{1}$, Gokalp Yildirir ${ }^{1}$, Matthew Villeneuve-Laroche ${ }^{1}$, Christophe

5 Roux ${ }^{7}$, Pierre-Marc Delaux ${ }^{7}$ and Nicolas Corradi ${ }^{1 \#}$

$6{ }^{1}$ Department of Biology, University of Ottawa. Ottawa, Canada.

$7 \quad 2$ Institute of Botany, The Czech Academy of Sciences. Průhonice, Czech Republic

$8{ }^{3}$ Institute of Experimental Botany, The Czech Academy of Science, Prague, Czech Republic

$9{ }^{4}$ Department of Biological Sciences, University of Toronto Scarborough, Toronto, Canada

$10{ }^{5}$ Department of Ecology and Evolutionary Biology, University of Toronto, Toronto, Canada

$11{ }^{6}$ Department of Microbiology \& Plant Pathology and Institute for Integrative Genome Biology,

12 University of California-Riverside, Riverside, California. USA

$13{ }^{7}$ Laboratoire de Recherche en Sciences Végétales, Université de Toulouse, UPS, CNRS 24

14 Chemin de Borde Rouge-Auzeville, Castanet-Tolosan, France

$15 *$ contributed equally

$16{ }^{\#}$ Lead contact

17 Corresponding authors

18 Nicolas Corradi email: ncorradi@uottawa.ca

19 Mathu Malar email: mmadhubioinfo@gmail.com

20 Nicolas Corradi Twitter: @Blunt_Microbe

21 Mathu Malar Twitter: @ madhubioinfo 


\section{Summary}

2 Arbuscular mycorrhizal fungi (AMF) (subphylum Glomeromycotina) [1] are amongst the most

3 prominent symbionts and form the Arbuscular Mycorrhizal symbiosis (AMS) with over 70\% of

4 known land plants [2] [3]. AMS allows plants to efficiently acquire poorly soluble soil nutrients

5 [4] and AMF to receive photosynthetically fixed carbohydrates. This plant-fungus symbiosis

6 dates back more than 400 million years [5], and is thought to be one of the key innovations that

7 allowed the colonization of lands by plants [6] . Genomic and genetic analyses in diverse plant

8 species started to reveal the molecular mechanisms that allowed the evolution of this symbiosis

9 on the host side, but how and when AMS abilities emerged in AMF remains elusive.

10 Comparative phylogenomics could be used to understand the evolution of AMS [7,8]. However,

11 the availability of genome data covering basal AMF phylogenetic nodes (Archaeosporales,

12 Paraglomerales) is presently based on fragmentary protein coding datasets [9]. Geosiphon

13 pyriformis (Archaeosporales) is the only fungus known to produce endosymbiosis with nitrogen-

14 fixing cyanobacteria (Nostoc punctiforme) presumably representing the ancestral AMF state [10-

15 12]. Unlike other AMF, it forms long fungal cells ('bladders') that enclose cyanobacteria. Once

16 in the bladder, the cyanobacteria are photosynthetically active and fix nitrogen, receiving

17 inorganic nutrients and water from the fungus. Arguably, G. pyriformis represents an ideal

18 candidate to investigate the origin of AMS and the emergence of a unique endosymbiosis. Here,

19 we aimed to advance knowledge in these questions by sequencing the genome of G. pyriformis,

20 using a re-discovered isolate.

21 Keywords: Geosiphon pyriformis, homokaryon, bladders, binning, metagenome, symbiosis,

22 cyanobacteria, Nostoc Punctiforme

\section{Results}

\section{General genome characteristics of Geosiphon pyriformis}

25 The only known culture of G. pyriformis was lost over a decade ago (A. Schüßler, pers. comm).

26 In an attempt to rediscover G. pyriformis, we searched and identified symbiotic bladders of the

27 G. pyriformis-N. punctiforme symbiosis (Figure 1) at the only known stable habitat of this

28 species in the Spessart Mountains near the village of Bieber in Germany [13]. 


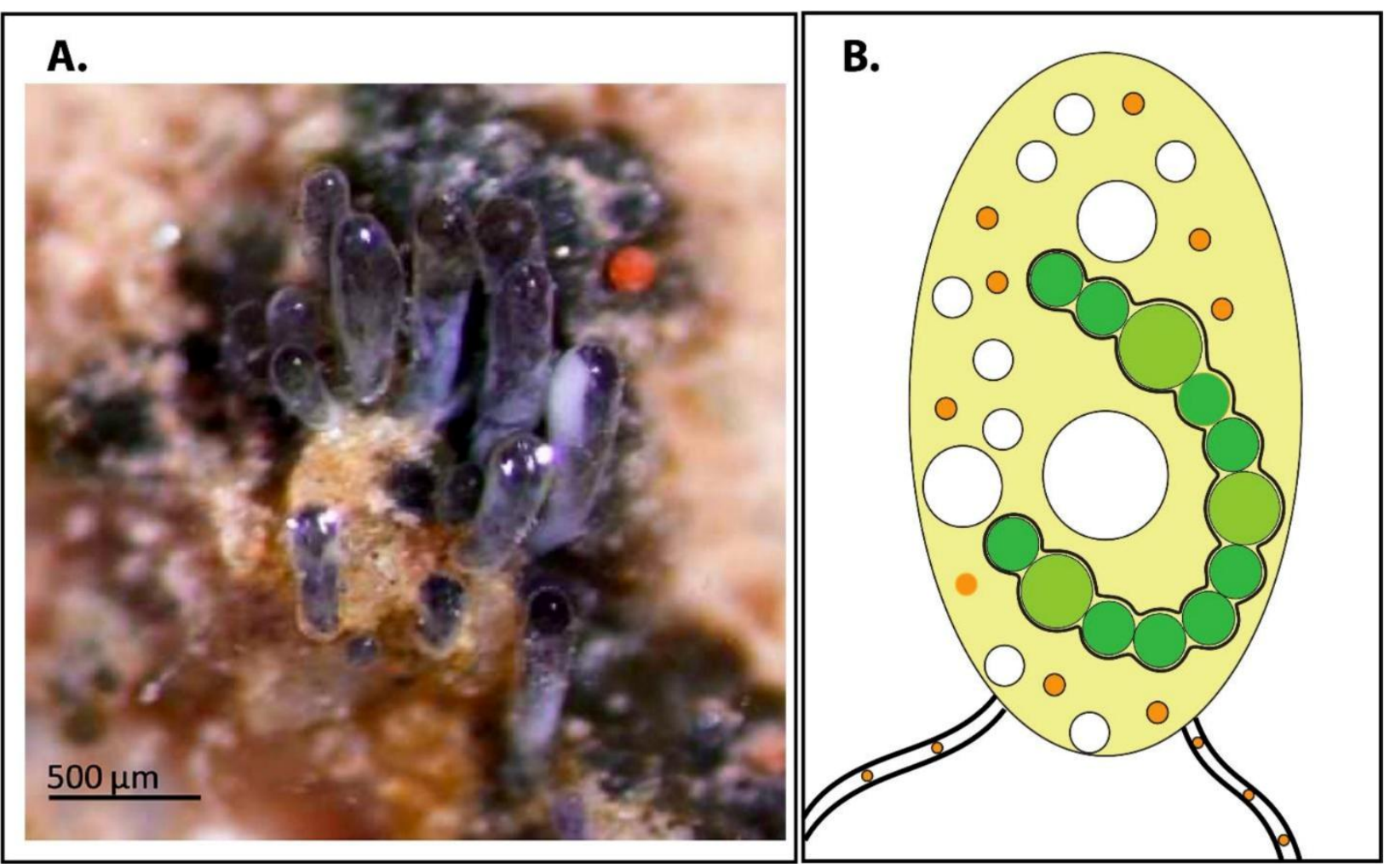

2 Figure 1: a. Image of G. pyriformis bladders in soil from its natural habitat in the Spessart mountains. b. Schematic representation of G. pyriformis bladders containing Nostoc cells (based on M. Kluge 2002). Nostoc cells are shown in dark green, and heterocysts (differentiated cell that carries out nitrogen fixation) are shown in light green. Bladders contain G. pyriformis nuclei (orange), and several vacuoles (white). Aseptate hyphae reach out and extend from the bladders.

7 Upon cultivation, we extracted DNA and RNA from active bladders. Total DNA was subjected 8 to $5 \mathrm{~Kb}$-mate-pair and 125 bp paired-end Illumina sequencing, producing respectively 47 and 81 9 million 125 bp paired ends and 5kb mate-pairs reads. G. pyriformis reads were identified using a 10 read binning approach recently implemented to assemble the genome of Diversispora epigea 11 epigaea [14], and upon identification these were assembled into a $129 \mathrm{MB}$ assembly and 795 12 scaffolds with an average read coverage of 118X. In parallel, total RNA was subjected to $150 \mathrm{bp}$ 13 paired-end Illumina sequencing. The resulting RNA-seq reads were mapped onto the $G$. 14 pyriformis genome assembly using STAR [15] and used for genome annotation after 15 implementing RepeatMasker [16]. This procedure identified of 24195 genes in G. pyriformis, 16 resulting in a BUSCO gene repertoire completeness of $96.2 \%$ (3.1\% complete duplicated). The 17 gene counts, estimated genome size, and genome statistics are all similar to those of model AMF 
1 species [17,18] and are indicative of high genome completeness (Table 1, see figure of "K-mer

2 distribution of filtered illumina genomic reads of G. pyriformis" in Data S1 and see table of 3 "Genome completeness using Busco" in Data S1). SignalP showed that, among all the genes

4 identified in G. pyriformis, 365 represent putative secreted proteins (see table of "Secretome

5 proteins from genomes" in Data S1) and 27\% of these are candidate effectors (see table of

6 "Effector prediction on G. Pyriformis genome "in Data S1). We also identified 19 putative 7 secreted CAZymes in G. pyriformis; in line with numbers found in other AMF species (Table S1 8 and Table $\mathrm{S} 2$ ).

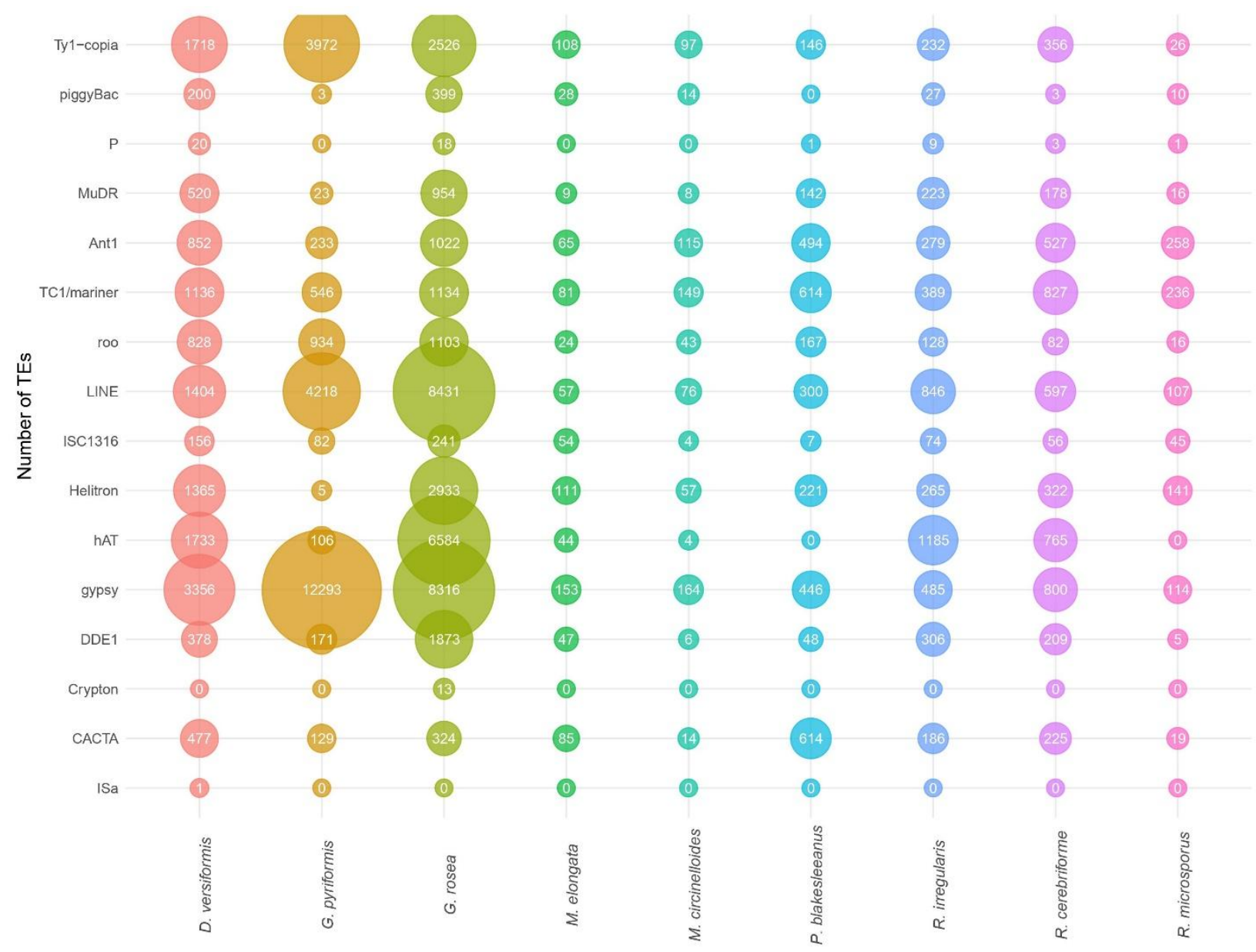

11 Figure 2: Bubble plot containing all transposable elements in the genomes of glomeromycotina 12 and mucoromycotina genomes used in this study. The figure shows the expansion of Gypsy 13 elements in G. pyriformis. 
2 AMF genomes carry a substantial fraction of transposable elements (TE) [18-21], and we found

3 that G. pyriformis has undergone similar TE expansions. The expansion of Gypsy transposable

4 elements in G. pyriformis is evident in comparison to all other AMF genomes (Figure 2). With

5 regards to TE, we find no evidence that G. pyriformis (see figure of "Heatmap showing evidence

6 for a "one-speed genome" in Geosiphon Pyriformis" in Data S1) carries a two-speed genome

7 [22]. Two-speed genomes are characterized by the presence of TE-poor and gene dense regions

8 that a clearly separate from others that contain rapidly evolving genomic regions that usually

9 carry less genes, abundant TE, and other repeat elements [22,23].

10 Using genome data and single nucleus data it was recently shown that AMF carry two genome

11 organizations - i.e., homokaryotic (co-existing nuclei carry one parental haploid genotype) or

12 dikaryotic (two parental genotypes co-exist in the mycelium) [21,24-26]. Mapping reads onto

13 the G. pyriformis genome revealed reduced levels of polymorphism $(0.5 \mathrm{SNP} / \mathrm{Kb})$ and allelic

14 frequencies suggesting that this species is carries low a low nuclear diversity and likely

15 homokaryotic (see figure of "Genome wide allele frequency of G. Pyriformis shows it is a

16 homokaryon" in Data S1).

\section{Placement of $G$. pyriformis based on phylogenomics}

18 The G. pyriformis genome annotation was used to identify the phylogenetic placement of this

19 species using amino-acid sequences. In this case, we used a set of 434 conserved fungal single

20 copy genes (data available at DOI: 10.5281/zenodo.1413687) to construct a phylogenetic tree of

21 the fungal kingdom. Phylogenomics supports the monophyly of Glomeromycotina and its close

22 relationship with Mortierellomycotina within the phylum Mucoromycota [1] (Figure 3). Within

23 Glomeromycotina, G. pyriformis groups within a monophyletic clade with Ambispora leptoticha

24 and Paraglomus occultum, which diverged around 287 MYA (Figure S1). This clade is distinct

25 from more diverged nodes that contain sequenced representatives from Glomerales and

26 Diversisporales [27]. The current placement of G. pyriformis as a sister lineage to A. leptoticha

27 and $P$. occultum has full statistical support, and is favored by $73 \%$ of the gene sequences we

28 used. Alternative topologies that place, for example, G. pyriformis at a basal node to all AMF

29 (Alt-T1;Figure S2, see table of "Results of alternative tree topology test for phylogenetic tree " in

30 Data S1) or as being associated with Glomerales or Diversisporales (Alt-T2 and Alt-T3; Figure 
1 S2,see table of "Results of alternative tree topology test for phylogenetic tree " in Data S1) were 2 all rejected significantly using statistical tests implemented in IQ-TREE, including the KH, SH, 3 ELW, and AU tests (see table of "Results of alternative tree topology test for phylogenetic tree " 4 in Data S1).

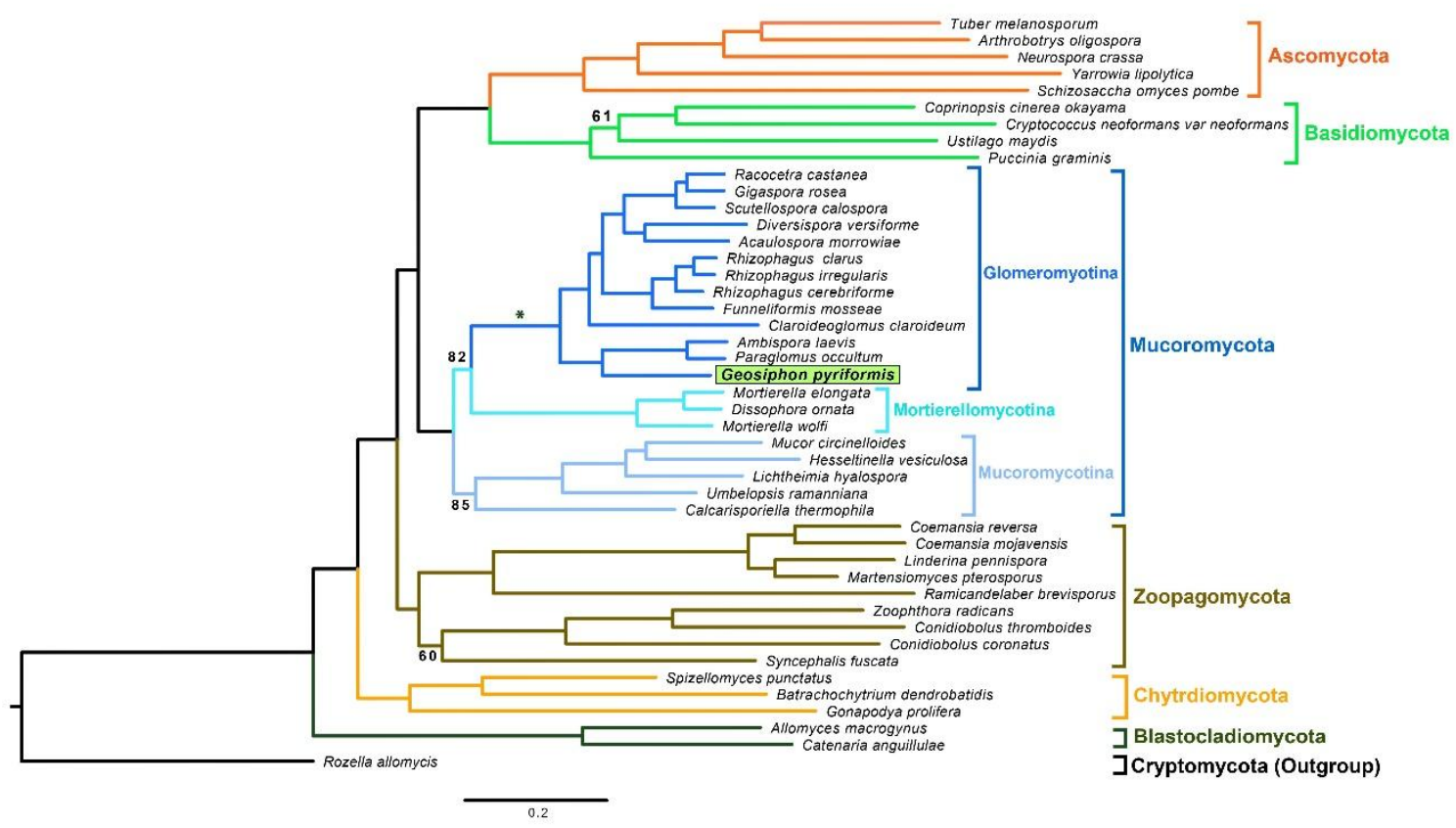

6 Figure 3: Phylogenetic tree representing the evolutionary relationships of fungi and placement of G. pyriformis in Glomeromycotina clade. The tree was resolved using maximum likelihood

8 phylogenetic reconstruction with IQ-TREE on a concatenated alignment of 434 protein coding

9 genes. Numbers indicate nodes with less than 100\% bootstrap support. Branches are coloured

10 according to their phylum.

11 Phylogenetic tests for all alternative topological placements of G. pyriformis were rejected. The

12 asterisk denotes the location where inferred gene losses and gains occurred in the MRCA of

13 Glomeromycotina.

14 The genome of Geosiphon pyriformis uncovers shared gene features in the Glomeromycotina

15 Phylogenomics revealed that G. pyriformis is a member of a clade that diverged early from the 16 lineage encompassing the already sampled Diversisporales and Glomerales. As such, the $G$. 17 pyriformis genome fills the gap in the genomic coverage of major AMF phylogenetic clades. 18 With this data in hand, we first searched for genetic features that arose in the most recent 
common ancestors (MRCA) of all Glomeromycotina by comparing orthogroups from five

2 available AMF genomes and four other members of the Mucoromycota as outgroups using

3 OrthoFinder [28]. This analysis identified 661 gains and 344 losses that occurred before the

4 divergence of the Glomeromycotina (Table S3 and see table of "Orthogroups lost in the MRCA

5 of Glomeromycotina" in Data S1).

6 Among the 344 orthogroups classified as lost in the MRCA of the Glomeromycotina, we note the

7 missing key enzymes involved in essential metabolic functions, such as sugar and thiamine

8 metabolisms, or in the biosynthesis of fatty acids. These genes are also referred to as "Missing

9 Glomeromycotina Core Genes" (MGCGs; see table of "Orthogroups lost in the MRCA of

10 Glomeromycotina" in Data S1, see table of "Missing MRCA genes across glomeromycotina core

11 genes" in Data S1), and our analysis reveals that these have also been lost by G. pyriformis.

12 Other key losses that affect all sequenced Glomeromycotina include enzymes that actively

13 degrade plant cell wall (see table of "Presence and absence of plant and fungal cell wall related

14 CAZymes" in Data S1). Among the 661 orthogroups gained in the MRCA, most encode for

15 proteins involved in signaling pathways (e.g., protein kinases), protein-protein interactions (e.g.,

16 the tetratricopeptide repeat, Sel1, the homodimerization BTB (Broad- Complex, Tramtrack and

17 Bric a brac), and WD- 40 domain- containing proteins) and High Mobility Box (HMG) (see

18 table of "Functions of genes which are gained in orthogroups" in Data S1).

19 Comparative genomics also showed that G. pyriformis carries the same signatures of sexual

20 reproduction found in AMF relatives. These include a complete set of meiosis-specific genes

21 (see table of "Identification of Meiosis specific genes" in Data S1) [29,30], and a highly

22 conserved genomic locus with architecture and sequence similarity to the mating-type (MAT)

23 locus of basidiomycetes [21,31,32] (see figure of "Transcriptional directions of the putative

24 AMF mating-type locus" in Data S1).

\section{Regulation of orthogroups gained in the MRCA of Glomeromycotina}

28 We investigated available gene expression data from the model AMF Rhizophagus irregularis, 29 and found that 7 and 39 orthogroups (with a total of 8 and 272 genes; see table of "Upregulation 
1 of ortholog genes from Rhiir2 genome "in Data S1 and see table of "Downregulation of

2 Orthogroups genes from Rhiir2" in Data S1) gained in the MRCA of Glomeromycotina are

3 respectively upregulated and downregulated across all four experimental conditions in the model

4 AMF Rhizophagus irregularis.

5 These conditions include symbiotic associations between $R$. irregularis and distinct plant hosts

6 (Medicago truncatula, Brachypodium distachyon ) [33-35], and others based on laser-capture

7 microdissection arbuscule-specific gene expression $[33,34,36]$. We investigated the putative

8 function of these differentially regulated genes by identifying protein motifs along their coding

9 sequences, and found that these are involved in a myriad putative cellular function, though these

10 mostly include protein tyrosine kinases, cytochrome p450, as well as FAD binding (see table of

11 "Upregulation of ortholog genes from Rhiir2 genome" and "Downregulation of Orthogroups

12 genes from Rhiir2" in Data S1). Lastly, one differentially regulated OG (OG0001728) shows

13 evidence of originating from horizontal gene transfer from bacteria - i.e., this orthogroup is

14 shared between bacteria and fungi (Figure S3).

\section{Distinct genomic features of Geosiphon pyriformis}

16 Besides the evolution of AMS, the acquisition of the G. pyriformis genome offers an opportunity

17 to identify innovations linked to the emergence of the only known cyanobacteria - fungus

18 endosymbiosis. To identify such innovations, hierarchical clustering and abundance of Pfam

19 domains was performed using available genomes in the Glomeromycotina and representatives of

20 Mucoromycotina and Mortierellomycotina (Figure S4). This analysis revealed a significant

21 overrepresentation of 16 protein domains in G. pyriformis compared to relatives in the

22 Mucoromycota - e.g., Lipase_3, RNase_H, Retrotrans gag domains, dUTPase, Spuma_A9PTase,

23 Myb_DNA-bind_6 (see table of "Pfam domain counts from genomes of mucoromycota used in

24 this study" and "p values calculated by fisher's test" in Data S1).

25 We also sought evidence of horizontal gene transfers (HGT) between partners of the unique

26 Geosiphon-Nostoc endosymbiosis, and found 18 genes with potential bacterial within in the $G$.

27 pyriformis genome (see table of "HGT containing genes with pfam domains" in Data S1).

28 Among putative HGT, two are protein encoding genes with significant sequence conservation

29 with Nostoc and Gamma proteobacteria homologues (see figure of "Phylogenetic tree showing

30 evidence of Horizontal gene transfer of Selenium binding protein" and "Phylogenetic tree 
1 showing evidence of horizontal gene transfer in Molybdenum cofactor carrier" in Data S1). All

2 putative HGT are located within contigs with average coverage and surrounded by genes of

3 AMF origin, suggesting these do not represent contaminants.

4 Discussion

5 MRCA of all extant Glomeromycotina carried hallmarks of mutualism and obligate biotrophy

6 Genome data from a representative of the basal node of the AMF phylogeny filled an important

7 gap in understanding the origin of AMS. Specifically, it allowed us to conclude that the MRCA

8 of all extant Glomeromycotina carried hallmarks of mutualism and obligate biotrophy - i.e., a

9 lack of genes for fatty acids and thiamine biosynthesis and nutrition, and a reduced number of

10 genes that actively degrade plant cells. As such, the mechanisms involved in AMS appeared

11 prior to the emergence of Glomeromycotina and a represent a synapomorphy of this sub-phylum.

12 G. pyriformis has also conserved genomic signatures of sexual reproduction, as well as an

13 apparent low nuclear polymorphism. Both traits are thus conserved across Glomeromycotina and

14 are in stark contrast with the notion that these organisms represent an ancient asexual lineage.

15 The retention of a conserved Glomeromycotina gene set in G. pyriformis, including a sub-set of 16 these involved in plant cell wall degradation, indicates that this species might be possible to form

17 mycorrhizae, although this has never been observed. As such, this retention could reflect an

18 intrinsic capacity for G. pyriformis to undergo classic (but rare) mycorrhizal associations with

19 plants under the right conditions. Although speculative at this point, this hypothesis is supported

20 by the identification of rare Geosiphon-like sequences in environmental samples [37-39].

\section{$21 \quad$ Novel symbiotic abilities and horizontal gene transfers in G. pyriformis}

22 As a result of losses in fatty acid biosynthesis genes, AMF are entirely dependent on the host 23 plant they associate with to obtain lipids [40-43]. Within this context, our findings suggest that,

24 during the switch from regular AMS to a fungal- cyanobacteria symbiosis, G. pyriformis has 25 evolved novel strategies to obtain lipids from its new host through the expansion of specific gene 26 motifs. Specifically, the G. pyriformis genome carries a striking over-representation of Lipase 3 27 protein domains that hydrolyze ester linkages of fatty acids. As Nostoc spp is known to produce 28 a wide variety of extracellular lipids in high amounts [40-43], it is possible that these abundant 29 lipids are released in the environment (like many other cellular compounds released by 
1 cyanobacteria [44-47]) and are then broken down by lipases to be used as an energy resource by

$2 \quad$ G. pyriformis.

3 As we find evidence of bacteria-like genes in the G. pyriformis genome, our work also suggests

4 that the co-existence of multiple endosymbionts and G. pyriformis nuclei within restricted

5 bladders offers some opportunities for horizontal gene exchange. Although none of the putative

6 bacterial genes we identified in G. pyriformis are functionally related, there is evidence that one

7 is differentially regulated during AMF symbiosis, supporting the notion that bacteria-like genes

8 can play a major role in fungal evolution $[14,48]$.

\section{Identification of a putative AMF core-symbiotic toolkit}

10 The G. pyriformis genome also enabled the identification of a putative core AMF symbiotic

11 toolkit conserved in all the sampled Glomeromycotina. This set of genes is differentially

12 regulated in model AMF during symbiotic interactions with different plant hosts, including

13 dicots, monocots and non-vascular plants, and thus provides a basis for future research on

14 symbiosis-related mechanisms in these plant symbionts. The identification of a core set of gene

15 gains specifically regulated during mycorrhizal symbiosis, and their conservation across the

16 Glomeromycotina phylogeny, also provides support for the early emergence of symbiosis-

17 specific gene functions in AMF over 400 million years ago, contemporaneously with the

18 evolution of the first land plants [49,50]. Lastly as genetic transformation is currently unfeasible

19 in Glomeromycotina, only assumptions can be proposed for the function of these putative core

20 genes. However, as some encode for chitin synthases, one attractive hypothesis could be that

21 some evolved for the production of short-chain chitooligosaccharides or lipo-

22 chitololigosaccharides that are known symbiotic signals triggering the activation of the symbiotic

23 program on the host plant $[2,51,52]$.

\section{Acknowledgements}

25 We thank Vasilis Kokkoris and Allison MacLean for comments on an earlier version of the

26 manuscript. N.C research is funded by the discovery program of the Natural Sciences and

27 Engineering Research council (RGPIN-2020-05643) and the Discovery Accelerator Supplements

28 program (RGPAS-2020-00033). N.C is a University of Ottawa Research chair in Microbial

29 Genomics. M.K. and C.K. were funded by the Czech Sciences Foundation (GAČR) as Junior

30 grant with the project number GJ16-16406Y. This work was also supported by the Agence 
1 Nationale de la Recherche (ANR) grant EVOLSYM (ANR-17-CE20-0006-01) to P.M.D, by the

2 Bill and Melinda Gates Foundation as Engineering the Nitrogen Symbiosis for Africa

3 (OPP1172165) to PM. D and by Laboratoire de Recherche en Sciences Végétales (LRSV)

4 laboratory, which belongs to the TULIP Laboratoire d'Excellence (ANR-10-LABX-41). J.E.S. is

5 a CIFAR Fellow in the program Fungal Kingdom: Threats and Opportunities. Y.W. and J.E.S.

6 were supported by US National Science Foundation grants DEB-1441715 and DEB-1557110.

7 Author Contributions

8 MMC, MK, CK, YW, JS, PM, and NC planned and designed the research, wrote the Manuscript

9 and helped with data analysis. MMC carried out genome annotation and bioinformatics analysis.

10 YW performed phylogenetic and molecular dating analysis. ECHC and GY produced repeat

11 analysis with MMC. JK performed ortholog analysis. CR performed the transcriptome analysis.

12 MVL helped improving the quality of images. MK and CK produced biological materials. NC

13 supervised all process.

\section{Declaration of Interests}

15 The authors declare no competing interests.

16 Star Methods

17 Resource availability

18 Further information and requests for resources and reagents should be directed to and will be

19 fulfilled by the Lead Contact, Nicolas Corradi (ncorradi@uottawa.ca).

20 Materials availability

21 This study did not generate new unique reagents.

\section{Experimental Model and Subject Details}

23 AM Fungi G. pyriformis were used for this study. Genome assembly is submitted in NCBI 24 accession number of AAOMT000000000.

\section{Data and Code Availability}

26 Genome assembly is available in NCBI with accession number of JAAOMT000000000. Genome 27 sequencing reads are submitted in SRA with accession number of SRR11466073, Bioproject 
PRJNA610605, Biosample SAMN14307302. RNA-seq reads are available in SRA with

2 accession of SRR12018969, SRR12018968, SRR12018970. All scripts used to analyze are

3 archived in https://github.com/madhubioinfo/Geosiphon.

5 METHOD DETAILS

6 Cultivation of $G$. pyriformis samples from natural habitat

7 G. pyriformis was sampled during autumn in the only known stable habitat near the village

8 Bieber in the Spessart (Germany). Active bladders of the Geosiphon-Nostoc endosymbiosis were

9 found in slightly acidic soil ( $\mathrm{pH}$ ). The bladders occurred close to the hornwort Anthoceros spp.

10 and the liverwort Blasia pusilla L., as these plants harbor the cyanobacteria needed to trigger the

11 Geosiphon-Nostoc endosymbiosis. After sampling spores and bladders were transferred to the

12 institute in Průhonice and cultured in beakers [53,54], which contain a small pot with a sterile

13 mixture of sand and soil (from the original habitat). The cultures were grown in a climate

14 chamber at $18^{\circ} \mathrm{C}$ with $14 \mathrm{~h}$ light and $10 \mathrm{~h}$ night. The substrate is kept wet by a filter paper, which

15 reaches from the substrate into a water reservoir in the beaker. To be maintained over time,

16 cyanobacteria be frequently added to the cultures. For our cultures, Nostoc punctiformis was

17 obtained from the Culture Collection of Algae (SAG) at the University of Göttingen (Germany)

18 as strain SAG69.79 [53,54].

\section{Genome and transcriptome sequencing and assembly}

20 High quality DNA was extracted from active bladders of G. pyriformis and Nostoc punctiforme

21 using the NucleoSpinII Plant kit (Machery-Nagel) and purified with the genomic DNA clean-up

22 kit (Machery-Nagel) using the manufactures recommendations. Total DNA was sent to Fasteris

23 (Switzerland) for library Illumina library preparation and sequencing using on 150 paired end

24 and $5 \mathrm{~kb}$ mate pairs inserts (illumina Nextera mate pair kit). Sequencing was performed using the

25 Illumina Hiseq 4000 platform. Total RNA was extracted using the RNeasy-Mini Kit (Qiagen) as

26 per instructions of the manufacturer for library RNA-seq Illumina library preparation with

27 sequencing of 150 cycles and paired ends.

28 Poor quality and adapter sequences were trimmed using Trimmomatic [55] with parameters the

29 following parameters of ILLUMINACLIP:2:30:10 SLIDINGWINDOW:5:20 LEADING:5 
TRAILING:5 MINLEN:50. The resulting $1 \mathrm{~GB}$ of non-redundant metagenome reads were

2 assembled using metaSPAdes V3.12.0 [56]. Assembled contigs were binned on the basis of tetra

3 nucleotide signature using CONCOCT [57], following part of the procedure used to assembly the

4 genome of Diversispora epigaea [14]. Binned clusters were annotated using BLAST v 2.6.0+

5 [58,59] and clusters containing bacterial hits were removed. Using this approach, 21 bona-fide

6 AMF clusters were retained, and used as reference to filter original paired-end and mate pair

7 reads with BLAT v. 36x1 [60]. The reads which had mapped to the filtered contigs and matched

8 by BLAT were then extracted to build cleaned sequence libraries that were assembled with

9 MaSuRCA 3.3.0 [61]. Additional round of nr BLAST searches on MaSuRCA assembled contigs

10 were performed to further remove contaminating bacteria. K-mer $(\mathrm{k}=21)$ based methods were

11 used on filtered reads to estimate genome size of G. pyriformis using jellyfish 1.1.12 [62] and

12 plotted in GenomeScope 2.0 [63] (see figure of "K-mer distribution of filtered illumina genomic

13 reads of G. pyriformis" in Data S1).

\section{Genome annotation}

15 Protein coding genes were predicted using Funannotate V1.7.4

16 (https://funannotate.readthedocs.io/) [DOI:10.5281/zenodo.3679386], which automates gene

17 prediction. Assembled transcripts using Trinity [64] and Rnaseq reads mapped bam file were

18 used as transcript evidence for gene call.

19 Transposable elements were predicted using TransposonPSI [65]. Repeat sequences were first

20 identified using RepeatModeler [66] with multiple numbers of iterations. The iteration with the

21 most number of repeats were then used for soft-masking the genome with REPEATMASKER

22 (open 4.0.646) [16]. Output files generated from above procedures were used to identify repeat

23 along the assembly. The completeness of genome assembly was assessed with BUSCO version

242.0 [67] with default parameters using the fungal gene dataset [fungi_odb9] ( see table of

25 "Genome completeness using Busco" in Data S1).

26 Putative gene functions were identified using Diamond BLASTX [59]. Pfam domain analysis 27 were performed using Pfamscan [68,69] (Figure S4) (see table "Pfam domain counts from 28 genomes of mucoromycota used in this study" and "pvalues calculated by fisher's test" in Data 29 S1) and Carbohydrate-active enzymes (CAZYme) were identified using the dbCAN CAZy 30 database [70] (Table S2) (see table of "Presence and absence of plant and fungal cell wall 
1 related CAZymes" in Data S1. Putative CAZymes were further verified through comparisons of

2 data from Morin et al 2019 [17] (Table S1). Secretory proteins were identified using previously

3 published pipelines [71,72] (see table of "Secretome proteins from genomes" in Data S1), and

4 effectors were identified using EffectorP 2.0 [73] ( see table of "effector prediction on $G$.

5 Pyriformis genome" in Data S1). The putative MAT loci of Paraglomus sp., and $R$. irregularis

6 were identified by BLAST search procedures (see figure "Transcriptional directions of the

7 putative AMF mating-type locus" and table "Identification of Meiosis specific genes" in Data

8 S1). The tests for a two-evolutionary rates analysis was performed in part by measuring

9 intergenic distance among genes in genome using a $\mathrm{R}$ script [22] ( see figure of "Heatmap

10 showing evidence for a "one speed genome" in Geosiphon Pyriformis" in Data S1). For this

11 study, published genomes of additional Glomeromycotina, Mortierellomycotina, and

12 Mucoromycotina were downloaded from JGI portal MycoCosm database [74,75] [DOI:

13 10.1093/nar/gkt1183].

\section{SNP calling and allele frequency analysis}

15 Filtered Mate Pair and Paired End reads were mapped onto the assembled G. pyriformis genome 16 using the BWA-MEM v 0.7.17 algorithm [76] and sorted into a BAM file using samtools (v 1.9)

17 [76]. Variants were called using FREEBAYES v1.2.0 [77] and filtered using vcftools [78].

18 Filtering cutoffs and procedures were as described by in Ropars et al. 2016 and Morin et al. 2019

$19[17,21]$ (see figure "Genome wide allele frequency of G. Pyriformis shows it is a homokaryon".

20 Quality filtered variants and SNPs which passed filtering were used for constructing allele

21 frequency plot using a custom R script (code available in GitHub repository).

22 Phylogenetic analysis and molecular dating

23 The phylogenomic analyses employed a set of 434 generally conserved and single-copy proteins

24 in fungi (data available at DOI: 10.5281/zenodo.1413687), which were developed through efforts

25 of the 1000 fungal genomes project and provided in the Joint Genome Institute MycoCosm site

$26[1,75,79]$. Profile-Hidden-Markov-Models of these markers were searched in the Geosiphon

27 predicted protein sequences using HMMER3 (v3.1b2) [68] and recovered 393 homologs (out of

28 the 434) in total. The 434 markers in 45 included fungal genomes were further collapsed into 57

29 partitions using a greedy search embedded in PartitionFinder v.2.1.1 for consistent phylogenetic

30 signals [80]. Phylogenetic trees were produced using the PHYling pipeline (data available DOI: 
10.5281/zenodo.1257002) and with maximum likelihood method implemented in IQ-TREE

2 (v.1.7-beta9) [81]. Concordance factors across the tree were calculated using the package

3 implemented in IQ-TREE.

4 The divergence time of Geosiphon sp. from the clade of "Ambispora leptoticha and Paraglomus

5 occultum" was estimated using the R8S v1.81 [82] with the phylogenetic tree reconstructed from

6 the earlier step. We employed five calibration constraints to calibrate the tree, including the

7 crown groups of Fungi (1100 MYA) [83], Dikarya (772 MYA) [83], Chytridiomycota (>573

8 MYA) [84-86], the MRCA of Chytridiomycota and terrestrial fungi (>750 MYA) [84-86], and

9 Glomeromycotina (>460 MYA) [11]. The divergence time of each clade was inferred using the

10 Langley-Fitch method with Powell algorithm [87-89].

\section{Alternative topology test and dating analyses}

12 To test the likelihood of other possible phylogenetic placements of G. pyriformis, we first

13 reconstructed the associated phylogenetic trees using constraint tree topology as illustrated in

14 Figure S2 via “-g” option of the IQTREE package (iqtree-1.7-beta9) [81] ( see table "Results of

15 alternative tree topology test for phylogenetic tree" in Data S1). We then compared our best tree

16 (shown as Figure 3) with alternative topologies to compute the log-likelihoods of the trees using

17 Kishino-Hasegawa test, Shimodaira-Hasegawa test, expected likelihood weight, and

18 approximately unbiased test via "-zb" and "-au" parameters in IQTREE [90-94]. All tests were

19 performed with 10,000 resampling estimated log-likelihood (RELL) method for reliable results.

20 The best-fit substitution models for the genome-scale data matrix were estimated using

21 ModelFinder implemented in IQTREE package [95].

\section{Detection of putative horizontal gene transfers}

23 To identify genes in Geosiphon that have potential origin in cyanobacteria, we compared the

24 Geosiphon sp. genome to the available fungal and cyanobacterial genomes. To highlight

25 potential HGT genes, we used a Python script (available in github repository) [96] to filter out

26 genome component in G. pyriformis with higher similarity score to cyanobacteria than any fungi,

27 excluding the G. pyriformis itself (see figure of "Phylogenetic tree showing evidence of

28 horizontal gene transfer in selenium binding protein" and "Phylogenetic tree showing evidence

29 of horizontal gene transfer in Molybdenum cofactor carrier" in Data S1) (see table of "HGT

30 containing genes with pfam domains" in Data S1). 


\section{Gene orthology and evolution of symbiotic specific genes}

2 Orthogroups resulting from the OrthoFinder run were parsed using a custom Python script. To be 3 retained, an orthogroup had to fill the following conditions: any sequence from the non-AMS

4 fungi, at least one sequence of Geosiphon pyriformis, one sequence of either Rhizophagus

5 irregularis or Rhizophagus cerebriforme and at least one sequence of Gigaspora rosea or 6 Diversispora epigaea (see table of "Functions of genes which are gained in orthogroups" in Data

7 S1). Reciprocally, orthogroups that could correspond to gene losses in the AMS fungi were 8 extracted by retaining orthogroups with no sequences of AMS fungi and at least one sequence of 9 each non-AMF fungi (Table S3) and (see table of "Orthogroups lost in the MRCA of 10 Glomeromycotina" and "Missing MRCA genes across glomeromycotina core genes" in Data $11 \mathrm{~S} 1)$.

12 Orthogroups showing evidence of regulation in symbiotic conditions in $R$. irregularis were 13 subjected to Maximum Likelihood (ML) analysis [92,97] to check for the absence of non-AMS

14 species. First, proteins contained in orthogroups were searched against the nine proteomes of 9 15 distinct species using the BLASTp+ v2.9.0 [98] with default parameters and an e-value threshold 16 fixed at 1e-05 (threshold was set to 1e-03 when no non-AMS species sequences were identified).

17 Then, proteins were aligned using MUSCLE v3.8.31 [99] with default parameters and resulting 18 alignment trimmed to remove positions with more than $80 \%$ of gaps using trimAl v1.4rev22 19 [100]. Prior to ML reconstruction, best fitting evolution model was tested using ModelFinder 20 [95] and then ML analysis was performed using IQ-TREE v1.6.1 [81] with 10,000 replicates of $21 \mathrm{SH}-a L R T$. Trees were visualized and annotated with the iTOL platform v5.5 [101,102] (Figure 22 S3).

23 After first round of phylogeny, orthogroups showing an AMF specific pattern were blasted 24 against the full MycoCosm database (1565 proteomes, last accessed: 03/01/2020) to confirm the 25 AMF-specific pattern and a phylogenetic analysis was performed following the procedure 26 described above.

\section{Differential expression analysis and combination of expression data to orthogroups}

28 Expression data of Rhizophagus irregularis in four conditions were used to select orthogroups

29 containing gene significantly deregulated in symbiosis for further analysis. Paired-end reads

30 were trimmed and fragments mapped onto Rhiir2_1 genome assembly of $R$. irregularis 
1 (https://genome.jgi.doe.gov/Rhiir2_1/). Stringent settings of mapping were used (similarity and

2 length read mapping criteria at $98 \%$ and $95 \%$, respectively). Genes differentially expressed

3 (DEG) in planta compared to extraradical mycelium were identified after EdgeR [103]

4 normalization with a false discovery rate (FDR) correction using CLC Genomic Workbench

5 (Qiagen). We retained genes showing an expression $>2$ - or $<-2$-fold times in planta compared to

6 extraradical hyphae (FDR $\leq 0.05$ ). Sets of 2683, 2518 and 2410 DEG were found in $M$.

7 truncatula, B. distachyon and L. cruciata respectively (see table "Upregulation of ortholog genes

8 from Rhiir2 genome" and "Downregulation of ortholog genes from Rhiir2 genome" in Data S1).

9 Detailed information on the data are available at the National Center for Biotechnology

10 Information (NCBI) Gene Expression Omnibus (GEO) portal (accession no GSE67926). The

11 analysis performed on RNA-seq data from arbuscocytes in M. truncatula [36] presented 6359

12 DEG.

\section{Quantification and Statistical analysis}

14 Statistical analysis and graphs were generated using R studio version 4.0.1 (2020-06-06)

\section{References}

16 1. Spatafora, J.W., Chang, Y., Benny, G.L., Lazarus, K., Smith, M.E., Berbee, M.L., Bonito, 17 G., Corradi, N., Grigoriev, I., Gryganskyi, A., et al. (2016). A phylum-level phylogenetic 18 classification of zygomycete fungi based on genome-scale data. Mycologia 108, 1028191046.

20 2. Parniske, M. (2008). Arbuscular mycorrhiza: The mother of plant root endosymbioses. $21 \quad$ Nat. Rev. Microbiol. 6, 763-775.

22 3. Brundrett, M.C. (2009). Mycorrhizal associations and other means of nutrition of vascular

25 4. Smith, S., and Read, D. (2008). Mycorrhizal Symbiosis.

26 5. Remy, W., Taylor, T.N., Hass, H., and Kerp, H. (1994). Four hundred-million-year-old 27 vesicular arbuscular mycorrhizae. Proc. Natl. Acad. Sci. U. S. A. 91, 11841-11843.

28 6. Strullu-Derrien, C., Selosse, M.A., Kenrick, P., and Martin, F.M. (2018). The origin and 29 evolution of mycorrhizal symbioses: from palaeomycology to phylogenomics. New 
Phytol. 220, 1012-1030.

27. Delaux, P.M., Radhakrishnan, G. V., Jayaraman, D., Cheema, J., Malbreil, M., Volkening,

J.D., Sekimoto, H., Nishiyama, T., Melkonian, M., Pokorny, L., et al. (2015). Algal ancestor of land plants was preadapted for symbiosis. Proc. Natl. Acad. Sci. U. S. A. 112, 13390-13395.

8. Radhakrishnan, G. V., Keller, J., Rich, M.K., Vernié, T., Mbadinga Mbadinga, D.L., Vigneron, N., Cottret, L., Clemente, H.S., Libourel, C., Cheema, J., et al. (2020). An ancestral signalling pathway is conserved in intracellular symbioses-forming plant lineages. Nat. Plants 6, 280-289.

9. Beaudet, D., Chen, E.C.H., Mathieu, S., Yildirir, G., Ndikumana, S., Dalpé, Y., Séguin, S., Farinelli, L., Stajich, J.E., and Corradi, N. (2018). Ultra-low input transcriptomics reveal the spore functional content and phylogenetic affiliations of poorly studied arbuscular mycorrhizal fungi. DNA Res. 25, 217-227.

10. Gehrig, H., Schüßler, A., and Kluge, M. (1996). Geosiphon pyriforme, a fungus forming endocytobiosis with Nostoc (cyanobacteria), is an ancestral member of the glomales: Evidence by SSU rRNA analysis. J. Mol. Evol. 43, 71-81.

11. Redecker, D. (2000). Glomalean Fungi from the Ordovician. Science 289, 1920-1921.

12. Schüßler, A., and Walker, C. (2011). 7 Evolution of the 'Plant-Symbiotic' Fungal Phylum, Glomeromycota. In Evolution of Fungi and Fungal-Like Organisms, pp. 163-185.

13. Schüßler, A., Schnepf, E., Mollenhauer, D., and Kluge, M. (1995). The fungal bladders of the endocyanosis Geosiphon pyriforme, a Glomus-related fungus: cell wall permeability indicates a limiting pore radius of only $0.5 \mathrm{~nm}$. Protoplasma 185, 131-139.

14. Sun, X., Chen, W., Ivanov, S., MacLean, A.M., Wight, H., Ramaraj, T., Mudge, J., Harrison, M.J., and Fei, Z. (2019). Genome and evolution of the arbuscular mycorrhizal fungus Diversispora epigaea (formerly Glomus versiforme) and its bacterial endosymbionts. New Phytol. 221, 1556-1573.

15. Dobin, A., Davis, C.A., Schlesinger, F., Drenkow, J., Zaleski, C., Jha, S., Batut, P., Chaisson, M., and Gingeras, T.R. (2013). STAR: Ultrafast universal RNA-seq aligner. Bioinformatics 29, 15-21. 
1 16. Smit, A.F.A., Hubley, R., and Green, P. (2010). RepeatMasker Open-3.0. 1996-2010. Inst. 2 Syst. Biol.

3 17. Morin, E., Miyauchi, S., San Clemente, H., Chen, E.C.H., Pelin, A., de la Providencia, I., 4 Ndikumana, S., Beaudet, D., Hainaut, M., Drula, E., et al. (2019). Comparative genomics 5 of Rhizophagus irregularis, R. cerebriforme, R. diaphanus and Gigaspora rosea highlights specific genetic features in Glomeromycotina. New Phytol. 222, 1584-1598.

18. Tisserant, E., Malbreil, M., Kuo, A., Kohler, A., Symeonidi, A., Balestrini, R., Charron, P., Duensing, N., Frei Dit Frey, N., Gianinazzi-Pearson, V., et al. (2013). Genome of an arbuscular mycorrhizal fungus provides insight into the oldest plant symbiosis. Proc. Natl. Acad. Sci. U. S. A. 110, 20117-20122.

19. Mathieu, S., Cusant, L., Roux, C., and Corradi, N. (2018). Arbuscular mycorrhizal fungi: intraspecific diversity and pangenomes. New Phytol. 220, 1129-1134.

20. Chen, E.C.H., Morin, E., Beaudet, D., Noel, J., Yildirir, G., Ndikumana, S., Charron, P., St-Onge, C., Giorgi, J., Krüger, M., et al. (2018). High intraspecific genome diversity in the model arbuscular mycorrhizal symbiont Rhizophagus irregularis. New Phytol. 220, 1161-1171. Available at: http://doi.wiley.com/10.1111/nph.14989 [Accessed September 9, 2019].

21. Ropars, J., Toro, K.S., Noel, J., Pelin, A., Charron, P., Farinelli, L., Marton, T., Krüger, M., Fuchs, J., Brachmann, A., et al. (2016). Evidence for the sexual origin of heterokaryosis in arbuscular mycorrhizal fungi. Nat. Microbiol. 1 .

22. Dong, S., Raffaele, S., and Kamoun, S. (2015). The two-speed genomes of filamentous pathogens: Waltz with plants. Curr. Opin. Genet. Dev. 35, 57-65.

23. Mathu Malar, C., Yuzon, J.D., Das, S., Das, A., Panda, A., Ghosh, S., Tyler, B.M., Kasuga, T., and Tripathy, S. (2019). Haplotype-phased genome assembly of virulent phytophthora ramorum isolate ND886 facilitated by long-read sequencing reveals effector polymorphisms and copy number variation. Mol. Plant-Microbe Interact. 32, 1047-1060.

24. Vandenkoornhuyse, P., Leyval, C., and Bonnin, I. (2001). High genetic diversity in arbuscular mycorrhizal fungi: Evidence for recombination events. Heredity (Edinb). 87, 243-253. 
1 25. Chen, E.C.C.H., Mathieu, S., Hoffrichter, A., Sedzielewska-Toro, K., Peart, M., Pelin, A.,

2 Ndikumana, S., Ropars, J., Dreissig, S., Fuchs, J., et al. (2019). Single nucleus sequencing

3 reveals evidence of inter-nucleus recombination in arbuscular mycorrhizal fungi. Elife 7 ,

$4 \quad 1-17$.

5 26. Croll, D., and Sanders, I.R. (2009). Recombination in Glomus intraradices, a supposed

6 ancient asexual arbuscular mycorrhizal fungus. BMC Evol. Biol. 9.

7 27. Krüger, M., Krüger, C., Walker, C., Stockinger, H., and Schüßler, A. (2012). Phylogenetic

8 reference data for systematics and phylotaxonomy of arbuscular mycorrhizal fungi from

9 phylum to species level. New Phytol. 193, 970-984.

1028 Emms, D.M., and Kelly, S. (2019). OrthoFinder: Phylogenetic orthology inference for 11 comparative genomics. Genome Biol. 20.

12 29. Corradi, N., and Lildhar, L. (2012). Meiotic genes in the arbuscular mycorrhizal fungi.

13 Commun. Integr. Biol. 5, 187-189.

14 30. Halary, S., Malik, S.B., Lildhar, L., Slamovits, C.H., Hijri, M., and Corradi, N. (2011).

15 Conserved meiotic machinery in Glomus spp., a putatively ancient asexual fungal lineage.

16 Genome Biol. Evol. 3, 950-958.

17 31. Idnurm, A., Walton, F.J., Floyd, A., and Heitman, J. (2008). Identification of the sex

18 genes in an early diverged fungus. Nature 451, 193-196.

19 32. Wong, S., Fares, M.A., Zimmermann, W., Butler, G., and Wolfe, K.H. (2003). Evidence

34. An, J., Zeng, T., Ji, C., de Graaf, S., Zheng, Z., Xiao, T.T., Deng, X., Xiao, S., Bisseling, from comparative genomics for a complete sexual cycle in the "asexual" pathogenic yeast Candida glabrata. Genome Biol. 4.

33. Gaude, N., Bortfeld, S., Duensing, N., Lohse, M., and Krajinski, F. (2012). Arbusculecontaining and non-colonized cortical cells of mycorrhizal roots undergo extensive and specific reprogramming during arbuscular mycorrhizal development. Plant J. 69, 510-528.

35. Zeng, T., Holmer, R., Hontelez, J., te Lintel-Hekkert, B., Marufu, L., de Zeeuw, T., Wu, 
F., Schijlen, E., Bisseling, T., and Limpens, E. (2018). Host- and stage-dependent secretome of the arbuscular mycorrhizal fungus Rhizophagus irregularis. Plant J. 94, 411425.

36. Fiorilli, V., Catoni, M., Miozzi, L., Novero, M., Accotto, G.P., and Lanfranco, L. (2009). Global and cell-type gene expression profiles in tomato plants colonized by an arbuscular mycorrhizal fungus. New Phytol.

37. Sheng, M., Chen, X., Zhang, X., Hamel, C., Cui, X., Chen, J., Chen, H., and Tang, M. (2017). Changes in arbuscular mycorrhizal fungal attributes along a chronosequence of black locust (Robinia pseudoacacia) plantations can be attributed to the plantation-induced variation in soil properties. Sci. Total Environ.

38. Berruti, A., Demasi, S., Lumini, E., Kobayashi, N., Scariot, V., and Bianciotto, V. (2017). Wild Camellia japonica specimens in the Shimane prefecture (Japan) host previously undescribed AMF diversity. Appl. Soil Ecol.

39. Krüger, C., Kohout, P., Janoušková, M., Püschel, D., Frouz, J., and Rydlová, J. (2017). Plant communities rather than soil properties structure arbuscular mycorrhizal fungal communities along primary succession on a mine spoil. Front. Microbiol.

40. Temina, M., Rezankova, H., Rezanka, T., and Dembitsky, V.M. (2007). Diversity of the fatty acids of the Nostoc species and their statistical analysis. Microbiol. Res. 162, 308321.

41. Patel, V.K., Sundaram, S., Patel, A.K., and Kalra, A. (2018). Characterization of Seven Species of Cyanobacteria for High-Quality Biomass Production. Arab. J. Sci. Eng.

42. López-Rosales, A.R., Ancona-Canché, K., Chavarria-Hernandez, J.C., Barahona-Pérez, F., Toledano-Thompson, T., Garduño-Solórzano, G., López-Adrian, S., Canto-Canché, B., Polanco-Lugo, E., and Valdez-Ojeda, R. (2019). Fatty acids, hydrocarbons and terpenes of nannochloropsis and nannochloris isolates with potential for biofuel production. Energies.

43. Steinhoff, F.S., Karlberg, M., Graeve, M., and Wulff, A. (2014). Cyanobacteria in Scandinavian coastal waters - A potential source for biofuels and fatty acids? Algal Res.

44. Rich, M.K., Nouri, E., Courty, P.E., and Reinhardt, D. (2017). Diet of Arbuscular Mycorrhizal Fungi: Bread and Butter? Trends Plant Sci. 22, 652-660. 
1 45. Luginbuehl, L.H., Menard, G.N., Kurup, S., Van Erp, H., Radhakrishnan, G. V.,

46. Cordeiro, R.S., Vaz, I.C.D., Magalhães, S.M.S., and Barbosa, F.A.R. (2017). Effects of

47. Keymer, A., Pimprikar, P., Wewer, V., Huber, C., Brands, M., Bucerius, S.L., Delaux,

48. Torres-Cortés, G., Ghignone, S., Bonfante, P., and Schüßler, A. (2015). Mosaic genome of P.M., Klingl, V., von Röpenack-Lahaye, E., Wang, T.L., et al. (2017). Lipid transfer from plants to arbuscular mycorrhiza fungi. Elife 6 . endobacteria in arbuscular mycorrhizal fungi: Transkingdom gene transfer in an ancient mycoplasma-fungus association. Proc. Natl. Acad. Sci. U. S. A. 112, 7785-7790.

49. Rensing, S.A. (2018). Great moments in evolution: the conquest of land by plants. Curr. Opin. Plant Biol. 42, 49-54.

50. Morris, J.L., Puttick, M.N., Clark, J.W., Edwards, D., Kenrick, P., Pressel, S., Wellman, C.H., Yang, Z., Schneider, H., and Donoghue, P.C.J. (2018). The timescale of early land plant evolution. Proc. Natl. Acad. Sci. U. S. A. 115, E2274-E2283.

51. Genre, A., Chabaud, M., Balzergue, C., Puech-Pagès, V., Novero, M., Rey, T., Fournier, J., Rochange, S., Bécard, G., Bonfante, P., et al. (2013). Short-chain chitin oligomers from arbuscular mycorrhizal fungi trigger nuclear $\mathrm{Ca} 2+$ spiking in Medicago truncatula roots and their production is enhanced by strigolactone. New Phytol. 198, 190-202.

52. Maillet, F., Poinsot, V., André, O., Puech-Pagés, V., Haouy, A., Gueunier, M., Cromer, L., Giraudet, D., Formey, D., Niebel, A., et al. (2011). Fungal lipochitooligosaccharide symbiotic signals in arbuscular mycorrhiza. Nature 469, 58-64.

53. Schüßler, A., and Wolf, E. (2005). Geosiphon pyriformis - a Glomeromycotan Soil Fungus Forming Endosymbiosis with Cyanobacteria. In In Vitro Culture of Mycorrhizas, pp. 271-289.

54. Mollenhauer, D., and Mollenhauer, R. (1988). Geosiphon cultures ahead. Endocytobios Cell Res 5, 69-73. 
1 55. Bolger, A.M., Lohse, M., and Usadel, B. (2014). Trimmomatic: A flexible trimmer for Illumina sequence data. Bioinformatics 30, 2114-2120.

56. Nurk, S., Meleshko, D., Korobeynikov, A., and Pevzner, P.A. (2017). MetaSPAdes: A new versatile metagenomic assembler. Genome Res. 27, 824-834.

5 57. Alneberg, J., Bjarnason, B.S., De Bruijn, I., Schirmer, M., Quick, J., Ijaz, U.Z., Lahti, L., Loman, N.J., Andersson, A.F., and Quince, C. (2014). Binning metagenomic contigs by coverage and composition. Nat. Methods 11, 1144-1146.

58. Altschul, S.F., Gish, W., Miller, W., Myers, E.W., and Lipman, D.J. (1990). Basic local alignment search tool. J Mol Biol 215, 403-410.

59. Buchfink, B., Xie, C., and Huson, D.H. (2014). Fast and sensitive protein alignment using DIAMOND. Nat. Methods 12, 59-60.

12 60. Kent, W.J. (2002). BLAT---The BLAST-Like Alignment Tool.

13 61. Zimin, A. V., Marçais, G., Puiu, D., Roberts, M., Salzberg, S.L., and Yorke, J.A. (2013). The MaSuRCA genome assembler. Bioinformatics 29, 2669-2677.

62. Marçais, G., and Kingsford, C. (2011). A fast, lock-free approach for efficient parallel counting of occurrences of k-mers. Bioinformatics 27, 764-770.

63. Vurture, G.W., Sedlazeck, F.J., Nattestad, M., Underwood, C.J., Fang, H., Gurtowski, J., and Schatz, M.C. (2017). GenomeScope: Fast reference-free genome profiling from short reads. In Bioinformatics, pp. 2202-2204.

64. Haas, B.J., Papanicolaou, A., Yassour, M., Grabherr, M., Blood, P.D., Bowden, J., Couger, M.B., Eccles, D., Li, B., Lieber, M., et al. (2013). De novo transcript sequence reconstruction from RNA-seq using the Trinity platform for reference generation and analysis. Nat. Protoc. 8, 1494-1512.

65. Hass, B. (2010). TransposonPSI: An application of PSI-Blast to mine (retro-)transposon ORF homologies. Broad Institute, Cambridge, MA, USA.

26 66. Smit, A., and Hubley, R. (2013). RepeatModeler. RepeatModeler.

27 67. Simão, F.A., Waterhouse, R.M., Ioannidis, P., Kriventseva, E. V., and Zdobnov, E.M. (2015). BUSCO: Assessing genome assembly and annotation completeness with single- 
copy orthologs. Bioinformatics 31, 3210-3212.

68. Hancock, J.M., Zvelebil, M.J., Hancock, J.M., and Bishop, M.J. (2004). HMMer. In Dictionary of Bioinformatics and Computational Biology.

69. Finn, R.D., Bateman, A., Clements, J., Coggill, P., Eberhardt, R.Y., Eddy, S.R., Heger, A., Hetherington, K., Holm, L., Mistry, J., et al. (2014). Pfam: The protein families database. Nucleic Acids Res.

70. Lombard, V., Golaconda Ramulu, H., Drula, E., Coutinho, P.M., and Henrissat, B. (2014). The carbohydrate-active enzymes database (CAZy) in 2013. Nucleic Acids Res. 42.

71. Kamel, L., Tang, N., Malbreil, M., San Clemente, H., Le Marquer, M., Roux, C., and dit Frey, N.F. (2017). The comparison of expressed candidate secreted proteins from two arbuscular mycorrhizal fungi unravels common and specific molecular tools to invade different host plants. Front. Plant Sci. 8.

72. Pellegrin, C., Morin, E., Martin, F.M., and Veneault-Fourrey, C. (2015). Comparative analysis of secretomes from ectomycorrhizal fungi with an emphasis on small-secreted proteins. Front. Microbiol. 6.

73. Sperschneider, J., Dodds, P.N., Gardiner, D.M., Singh, K.B., and Taylor, J.M. (2018). Improved prediction of fungal effector proteins from secretomes with EffectorP 2.0. Mol. Plant Pathol. 19, 2094-2110.

74. Grigoriev, I. V., Nordberg, H., Shabalov, I., Aerts, A., Cantor, M., Goodstein, D., Kuo, A., Minovitsky, S., Nikitin, R., Ohm, R.A., et al. (2012). The Genome Portal of the Department of Energy Joint Genome Institute. Nucleic Acids Res. 40.

75. Grigoriev, I. V., Nikitin, R., Haridas, S., Kuo, A., Ohm, R., Otillar, R., Riley, R., Salamov, A., Zhao, X., Korzeniewski, F., et al. (2014). MycoCosm portal: Gearing up for 1000 fungal genomes. Nucleic Acids Res. 42.

76. Li, H., and Durbin, R. (2009). Fast and accurate short read alignment with BurrowsWheeler transform. Bioinformatics 25, 1754-1760.

77. Garrison, E., and Marth, G. (2012). Haplotype-based variant detection from short-read sequencing. arXiv Prepr. arXiv1207.3907. Available at: http://arxiv.org/abs/1207.3907. 
1 78. Danecek, P., Auton, A., Abecasis, G., Albers, C.A., Banks, E., DePristo, M.A.,

2 Handsaker, R.E., Lunter, G., Marth, G.T., Sherry, S.T., et al. (2011). The variant call

3 format and VCFtools. Bioinformatics 27, 2156-2158.

4 79. Stajich, J.E. (2017). Fungal Genomes and Insights into the Evolution of the Kingdom. The 5 Fungal Kingdom, 619-633.

6 80. Lanfear, R., Calcott, B., Ho, S.Y.W., and Guindon, S. (2012). PartitionFinder: Combined

81. Nguyen, L.T., Schmidt, H.A., Von Haeseler, A., and Minh, B.Q. (2015). IQ-TREE: A fast

82. Sanderson, M.J. (2003). r8s: Inferring absolute rates of molecular evolution and divergence times in the absence of a molecular clock. Bioinformatics 19, 301-302.

83. Parfrey, L.W., Lahr, D.J.G., Knoll, A.H., and Katz, L.A. (2011). Estimating the timing of early eukaryotic diversification with multigene molecular clocks. Proc. Natl. Acad. Sci. U. S. A. $108,13624-13629$.

84. Lutzoni, F., Nowak, M.D., Alfaro, M.E., Reeb, V., Miadlikowska, J., Krug, M., Arnold, A.E., Lewis, L.A., Swofford, D., Hibbett, D., et al. (2018). Contemporaneous radiations of fungi and plants linked to symbiosis. Nat. Commun. 9, 1-11.

85. Wang, Y., White, M.M., and Moncalvo, J.-M.M. (2019). Diversification of the gut fungi Smittium and allies (Harpellales) co-occurred with the origin of complete metamorphosis of their symbiotic insect hosts (lower Diptera). Mol. Phylogenet. Evol. 139, 106550.

86. Chang, Y., Wang, S., Sekimoto, S., Aerts, A., Choi, C., Clum, A., LaButti, K., Lindquist, E., Ngan, C.Y., Ohm, R.A., et al. (2015). Phylogenomic analyses indicate that early fungi evolved digesting cell walls of algal ancestors of land plants. Genome Biol. Evol. 7, 15901601.

87. Langley, C.H., and Fitch, W.M. (1974). An examination of the constancy of the rate of molecular evolution. J. Mol. Evol. 3, 161-177. 
1 88. Gill, P.E., Murray, W., and Wright, M.H. (1981). Practical optimization (New York:

$2 \quad$ Academic Press).

3 89. Press, W.H., Flannery, B.P., Teukolsky, S.A., and Vetterling, W.T. (1992). Numerical

4 recipes in C 2nd ed. (New York: Cambridge University Press).

5 90. Shimodaira, H. (2002). An approximately unbiased test of phylogenetic tree selection.

$6 \quad$ Syst. Biol. 51, 492-508.

7 91. Shimodaira, H., and Hasegawa, M. (1999). Multiple comparisons of log-likelihoods with

8 applications to phylogenetic inference. Mol. Biol. Evol. 16, 1114-1116.

9 92. Kishino, H., Miyata, T., and Hasegawa, M. (1990). Maximum likelihood inference of 10 protein phylogeny and the origin of chloroplasts. J. Mol. Evol. 31, 151-160.

11 93. Kishino, H., and Hasegawa, M. (1989). Evaluation of the maximum likelihood estimate of 12 the evolutionary tree topologies from DNA sequence .... J. Mol. Evol. 29, 170-179.

13 94. Strimmer, K., and Rambaut, A. (2002). Inferring confidence sets of possibly misspecified 14 gene trees. Proc. R. Soc. B Biol. Sci. 269, 137-142.

15 95. Kalyaanamoorthy, S., Minh, B.Q., Wong, T.K.F., Von Haeseler, A., and Jermiin, L.S. 16 (2017). ModelFinder: fast model selection for accurate phylogenetic estimates. Nat. 17 Methods 14, 587-589.

18 96. Wang, Y., White, M.M., Kvist, S., and Moncalvo, J.M. (2016). Genome-Wide Survey of Gut Fungi (Harpellales) Reveals the First Horizontally Transferred Ubiquitin Gene from a Mosquito Host. Mol. Biol. Evol. 33, 2544-2554.

$2197 . \quad$ Guindon, S., Dufayard, J.F., Lefort, V., Anisimova, M., Hordijk, W., and Gascuel, O. (2010). New algorithms and methods to estimate maximum-likelihood phylogenies: Assessing the performance of PhyML 3.0. Syst. Biol. 59, 307-321.

24 98. Camacho, C., Coulouris, G., Avagyan, V., Ma, N., Papadopoulos, J., Bealer, K., and

99. Edgar, R.C. (2004). MUSCLE: Multiple sequence alignment with high accuracy and high throughput. Nucleic Acids Res. 32, 1792-1797. 
automated alignment trimming in large-scale phylogenetic analyses. Bioinformatics 25 , 1972-1973.

3 101. Letunic, I., and Bork, P. (2007). Interactive Tree Of Life (iTOL): An online tool for phylogenetic tree display and annotation. Bioinformatics 23, 127-128.

5 102. Letunic, I., and Bork, P. (2019). Interactive Tree of Life (iTOL) v4: Recent updates and new developments. Nucleic Acids Res. 47.

7 103. Robinson, M.D., McCarthy, D.J., and Smyth, G.K. (2009). edgeR: A Bioconductor package for differential expression analysis of digital gene expression data. Bioinformatics 26, 139-140.

14 Table 1: Summary statistics for genome assembly of sequenced G. pyriformis and other 15 species from Glomeromycotina and selected Mucoromycota used in this study

\begin{tabular}{|l|l|l|l|l|l|l|l|l|}
\hline \multicolumn{1}{|c|}{ Genomes } & $\begin{array}{c}\text { Assembly } \\
\text { size }\end{array}$ & $\begin{array}{c}\text { No of } \\
\text { scaffolds }\end{array}$ & $\begin{array}{c}\text { Scaffold } \\
\text { N50 }\end{array}$ & $\begin{array}{c}\text { Largest } \\
\text { scaffold } \\
\text { (Kb) }\end{array}$ & $\begin{array}{c}\text { Total } \\
\text { Gap\% }\end{array}$ & $\begin{array}{c}\text { Repeat } \\
\%\end{array}$ & $\begin{array}{c}\text { Busco } \\
\text { completeness } \\
\%\end{array}$ & $\begin{array}{c}\text { GC } \\
\%\end{array}$ \\
\hline $\begin{array}{l}\text { Geosiphon } \\
\text { Pyriformis }\end{array}$ & 129 & 795 & 703 & 2733.91 & 0.023 & 64.35 & 96.2 & 29.25 \\
\hline $\begin{array}{l}\text { Gigaspora } \\
\text { rosea V1.0 }\end{array}$ & 597.95 & 7526 & 734 & 1204.75 & 7.92 & 63.44 & 97.9 & 28.81 \\
\hline $\begin{array}{l}\text { Rhizophagus } \\
\text { ceribriforme } \\
\text { DAOM227022 } \\
\text { V1.0 }\end{array}$ & 136.89 & 2592 & 266 & 709.02 & 17.60 & 24.77 & 98.3 & 26.55 \\
\hline
\end{tabular}




\begin{tabular}{|c|c|c|c|c|c|c|c|c|}
\hline $\begin{array}{l}\text { Rhizophagus } \\
\text { irregularis } \\
\text { DAOM } \\
197198 \text { V2.0 }\end{array}$ & 136.80 & 1123 & 129 & 1375.86 & 5.06 & 26.38 & 98 & 27.53 \\
\hline $\begin{array}{l}\text { Diversispora } \\
\text { versiformis } \\
\text { strain IT104 }\end{array}$ & 147 & 731 & 434 & 2010.39 & 0.061 & 43.6 & 98.2 & 25.1 \\
\hline $\begin{array}{l}\text { Rhizopus } \\
\text { microsporus } \\
\text { ATCC11559 } \\
\text { V1 }\end{array}$ & 25.97 & 131 & 8 & 2782.17 & 2.41 & 4.68 & 98.6 & 37.48 \\
\hline $\begin{array}{l}\text { Mucor } \\
\text { Circinelloides } \\
\text { CBS } 277.49 \\
\text { V2.0 }\end{array}$ & 36.59 & 26 & 4 & 6050.25 & 0.00 & 20.38 & 97.2 & 42.17 \\
\hline $\begin{array}{l}\text { Phycomyces } \\
\text { Blakesleeanus } \\
\text { NRRL1555 } \\
\text { V2.0 }\end{array}$ & 53.94 & 80 & 11 & 4452.46 & 1.06 & 9.74 & 96.9 & 35.78 \\
\hline $\begin{array}{l}\text { Mortierella } \\
\text { Elongata } \\
A G-77\end{array}$ & 49.86 & 473 & 31 & 1526.29 & 0.30 & 4.63 & 99.7 & 48.05 \\
\hline
\end{tabular}


